\title{
Impact of Targeted Scanning Protocols on Perinatal Outcomes in Pregnancies at Risk of Placenta Accreta Spectrum or Vasa Previa
}

\author{
Yaakov MELCER ${ }^{1}, M D ;$ Eric JAUNIAUX²,MD,PhD; Shlomit MAYMON ${ }^{1}$; Anna \\ TSVIBAN ${ }^{1}, M D$; Marina PEKAR-ZLOTIN ${ }^{1}, M D$; Moshe BETSER ${ }^{1}, M D$; Ron \\ MAYMON ${ }^{1}, M . D$. \\ ${ }^{1}$ Department of Obstetrics and Gynecology, Assaf Harofeh Medical Center, Zerifin, \\ Israel, affiliated with the Sackler School of Medicine, Tel-Aviv University, Tel-Aviv, \\ Israel; ' 2 Academic Department of Obstetrics and Gynaecology, EGA Institute for \\ Women's Health, Faculty of Population Health Sciences, University College \\ London (UCL), London, UK
}

No funding was obtained for this study.

The authors report no conflict of interest.

*Corresponding author: Ron Maymon MD, Department of Obstetrics and Gynecology, Assaf Harofeh Medical Center, Zerifin, 70300, Israel. Telephone: +972-8-9779695, Fax: +972-8-9779089, E-mail: maymonrb@bezeqint.net

Word count: Abstract $=305$ \& Main text $=2795$ 
Condensation: Implementation of prenatal targeted scanning for placenta accreta spectrum and vasa previa in pregnant women at risk improves both maternal and neonatal obstetric outcomes.

Short title: Impact of targeted scanning on placenta accreta spectrum and vasa previa.

\section{Implications and Contributions}

- This study was conducted to evaluate the impact of targeted scanning of placenta accreta syndrome and vasa previa on perinatal outcomes.

- Targeted scanning improves maternal and neonatal outcomes for placenta accreta syndrome and vasa previa, respectively.

- Adding targeted scanning for placenta accreta syndrome and vasa previa to routine antenatal ultrasound examinations is feasible. 


\section{ABSTRACT}

Background: Placenta accreta spectrum (PAS) and vasa previa (VP) are congenital disorders of placentation associated with high morbidity and mortality for both mothers and newborns when undiagnosed before delivery. Prenatal diagnosis of these conditions is essential to allow multidisciplinary management and thus improve perinatal outcomes.

Objectives: To compare perinatal outcome in women with PAS or VP before and after implementation of targeted scanning protocols.

Methods: This retrospective study included two non-concurrent cohorts for each condition before and after implementation of the corresponding protocols (20041012 versus $2013-2016$ for PAS and 1988-2007 versus 2008-2016 for VP). Clinical reports of women diagnosed with PAS and VP during the study periods were reviewed and outcomes were compared.

Results: In total, there were 97 cases of PAS and 51 cases with VP, all confirmed at delivery. In both cohorts, the prenatal detection rate increased after implementation of the s scanning protocols (28/65 (43.1\%) cases versus $31 / 32$ (96.9\%) cases; $p<0.001$ for PAS and $9 / 18(50 \%)$ cases versus $29 / 33(87.9 \%)$ cases, $87.9 \%$; $p<0.01$ for VP). The perinatal outcome improved also significantly in both cohorts after implementation of the protocols. In PAS cohort, the estimated blood loss and the postoperative hospitalization stay decreased between periods ( $1520 \pm 845$ versus $1168 \pm 707 \mathrm{ml}, \mathrm{p}<0.01$ and $10.9 \pm 14.1$ versus $5.7 \pm 2.2$ days, $p<0.05$, respectively). In VP cohort, the number of 5 minute Apgar score $\leq 5$ and 
umbilical cord $\mathrm{pH}<7$ decreased between periods (5/18 (27.8\%) cases versus $1 / 33$ $(3 \%)$ cases; $p<0.05)$ and $4 / 18(22.2 \%)$ cases versus $1 / 33(3 \%)$ cases; $p<0.05$, respectively).

Conclusions: The implementation of standardized prenatal targeted scanning protocols for pregnant women with risk factors for PAS and VP was associated with improved maternal and neonatal outcomes. The continuous increases in the rates of caesarean deliveries and used of assisted reproductive technology highlights the need to develop training programs and introduce targeted scanning protocols at the national and international levels.

Key words: implementation; maternal morbidity; newborn morbidity; obstetric outcome; placental accrete spectrum; perinatology; target scan; ultrasound; vasa previa 


\section{INTRODUCTION}

Congenital disorders of placental and umbilical cord development include mainly anomalies of placental shape, location and accreta placentation and velamentous cord insertion and abnormal vasculature. ${ }^{1}$ Placental location and the number of umbilical vessels are routinely screen for at the mid-gestation detailed fetal anatomy scan, around the world. By contrast, placenta accreta spectrum (PAS) and vasa previa (VP) which are associated with high maternal and neonatal morbidity and mortality ${ }^{2}$ are not recommended to be screen for by specialist organizations including by the American College of Obstetricians and Gynecologists (ACOG), the American Institute of Ultrasound in Medicine (AIUM) and Royal College of Obstetricians and Gynaecologists (RCOG). ${ }^{3-8}$ Although targeted screening of high risk pregnancies has been discussed ${ }^{9-12}$, it has not been implemented at national or international level mainly due to limited supporting data but also lack of resources and training programs in many countries. ${ }^{12}$

PAS is defined as the abnormal adherence to or invasion of the villous tissue into the myometrium secondary to damage to the endometrium-myometrial interface of the uterine wall. ${ }^{1,13}$ Although, theoretically any damages to the uterine wall can lead to accreta placentation, the main cause of PAS is the uterine scar of a prior caesarean delivery $(C D)$, the incidence of which has increased exponentially worldwide in the last two decades. ${ }^{2,12}$ Adherent and invasive placentation may co-exist in the same placental bed and may evolve with advancing gestation. ${ }^{13}$ When unsuspected at the time of delivery, attempts to 
manually remove a placenta accreta typically provoke massive haemorrhage leading to high maternal morbidity and mortality. ${ }^{2}$ The prenatal ultrasound diagnosis of PAS is highly accurate in specialist units, in particular when associated with an anterior placenta previa or low-lying placenta. ${ }^{12,14}$

VP occurs when fetal vessels run through the membranes, cross the internal os of cervix under the fetal presentation, either to reach a velamentous cord (type I) or to connect the main placenta with a succenturiate or accessory lobe (type II). ${ }^{1,2}$ Being unprotected by the chorionic plate or Wharton's jelly of the umbilical cord, VP are liable to rupture either in active labour or at amniotomy which can be rapidly fatal for the fetus. A recent systematic review of the incidence and risk factors of vasa praevia including 13 studies reporting on 569410 women has indicated that $83 \%$ of the 325 cases reviewed presented with one or more risk factors, including placenta praevia, bilobed placenta, succenturiate placental lobes, conception by ART and velamentous cord insertion $(\mathrm{VCl}) .{ }^{15} \mathrm{~A}$ recent populationbased cohort study has shown that $95 \%$ of the 58 women diagnosed prenatally with VP had at least one risk factor with VCl (62\%) and low-lying placenta (60\%) the most prevalent. ${ }^{16}$ Twin pregnancies are at higher risks of $\mathrm{VCl}$ and VP and in vitro fertilization (IVF) is an additional risk factor of abnormal cord insertion and VP. ${ }^{16}$ The prenatal ultrasound diagnosis of VP using transvaginal sonography (TVS) and colour Doppler imaging (CDI) is highly accurate and specific. ${ }^{2,17}$

There is increasing evidence that multidisciplinary team (MDT) management of pregnancies with suspected PAS is superior to standard obstetric care ${ }^{18,19}$ and prenatal diagnosis of VP has been shown to improve the case perinatal survival 
rates to $95 \% .{ }^{16,21}$ We have developed targeted scanning protocols for both PAS and VP including clinical risk factors and ultrasound scoring system for PAS. ${ }^{22}$ The aims of the present study was to evaluate the perinatal outcome in women with PAS or VP before and after implementation of targeted scanning protocols.

\section{MATERIAL AND METHODS}

This study included two non-concurrent cohorts of pregnancies complicated by PAS or VP in a single-centre. In all cases, the diagnosis was confirmed at birth using clinical criteria similar to those proposed by Collins et $\mathrm{al}^{23}$ for PAS or standard histopathological examination features for both PAS and VP. ${ }^{1}$ In cases of PAS, detailed microscopic reports were only obtained in cases that underwent partial myometrial resection or caesarean hysterectomy. Women who did not deliver at our centre were excluded from the analysis. This study was approved by our Institutional Review Board (\#238-16).

Targeted scanning protocols were introduced in our antenatal clinics in 2013 for PAS and in 2008 for VP. Before 2013, pregnant patients diagnosed with PAS were managed on a case-by-case basis without MDT care. In 2013, we developed a clinical protocol to scan women with at least one previous $C D$ using ultrasound based scoring system as previously described ${ }^{22}$ and to manage them using an MDT approach. In brief, all women were assessed using a scoring system based on the following: number and size of placental lacunae; obliteration of the demarcation between the uterus and placenta; placental location; color Doppler signals within placental lacunae; 
hypervascularity of the placenta-bladder and/or uteroplacental interface zone; and number of previous CD. Each criteria was assigned 0, 1 or 2 points and the sum of points yielded the final score. Patients were classified into low ( $\leq 5$ points), moderate (6-7 points) or high (8-12 points) probability for PAS based on the final score. All pregnant women diagnosed with or suspected of PAS were provided with MDT perinatal care including follow-up until delivery be experienced ultrasonographers and obstetricians and review before delivery by neonatologists, gynaecological surgeons, haematologists and anaesthesiologists. Preoperative ultrasound mapping of the placental location was performed to assist in determining the optimal approach to abdominal wall entry and uterine incisions to avoid the placenta before delivery of the fetus. Delivery was planned between 35-37 weeks, depending on maternal symptoms. When gross placental invasion is evident at surgery, when major bleeding occur during manual delivery, when the placenta cannot be detached from the uterine wall or when part of the placenta remains attached, it is common practice in our institute to stop attempting to remove the placenta manually and to place a B-Lynch compressive suture or to perform an emergency caesarean hysterectomy if the bleeding is not controlled. The presence and severity of placental invasion was determined by the surgeons and the corresponding clinical data were documented in the electronic file of the patient.

Similarly, in 2008, we introduced a targeted ultrasound scanning protocol in pregnancies at risk for VP. ${ }^{24}$ The sonographic diagnoses of VP were all conformed by transvaginal sonography combined with CDI as previously described. ${ }^{24}$ Briefly, the VP were visualized in gray-scale sonography as parallel or circular echogenic lines within $2 \mathrm{~cm}$ from the internal cervical os, while color Doppler showed these 
structures to be vessels, and pulsed Doppler revealed a fetal arterial or venous waveform. From 2008, all asymptomatic women presenting with VP were followedup with transvaginal ultrasound for cervical length and VP position every 2 weeks from the time of the first diagnosis of VP until delivery. ${ }^{17}$ The timing of delivery was scheduled according to changes in cervical length and/or clinical symptoms (mainly uterine contractions and/or vaginal bleeding), following a course of corticosteroids. When the cervical remains stable with normal fetal development and there are no clinical symptoms delivery was planned at 35-36 weeks' gestation. ${ }^{17}$

Before implementation of the scanning protocols, cases had to be recovered from medical records whereas all cases post-implementation of the protocols were recorded contemporaneously in a database. Data collected included demographics, obstetrical history, surgical findings, intraoperative and postoperative maternal complication and care. Information collected from neonatal medical records included Apgar score, cord blood gases $\mathrm{pH}$, need for resuscitation and intubation, blood products transfusion, admission to neonatal intensive care unit, duration of hospital stay and perinatal mortality. Perinatal outcome and before and after implementation (2004-2012 versus 2013-2016 for PAS and 1988-2007 versus 2008-2016 for VP) of the corresponding targeted scanning protocols were compared.

\section{Statistical analysis}

Statistical analysis was performed by the statistical laboratory at Tel Aviv University using SPSS software (SPSS Inc., version 24 Chicago, IL, USA). Descriptive 
variables are presented as mean \pm standard deviation. Frequencies are presented as percentages. The Student's $t$-test, Pearson Chi-Square test, Fisher's exact test, and the Mann-Whitney rank test were used to compare the selected parameters. Correlations between CD and IVF during the study period were performed using the Pearson coefficient. A p value of $<0.05$ was considered statistically significant.

\section{RESULTS}

The search of our database identified 97 pregnancies diagnosed with PAS and 51 with VP. Figure 1 presents the demographics of our obstetric population since 1988. Overall, the number of deliveries has increased during the study period (Figure 1A). There was a significant increase in the percentages of both $C D$ $\left(r^{2}=0.96 ; p<0.0001\right)\left(\right.$ Figure 1B) and pregnancies resulting from IVF $\left(r^{2}=0.94\right.$; $p<0.0001)$ (Figure 1C).

Table 1 and Figure 2 describes and compares the clinical characteristics of the cases diagnosed with PAS before and after the introduction of the scanning protocol. The prenatal detection rate of PAS was significantly higher after the introduction of the protocol (28/65 cases, $43.1 \%$ versus $31 / 32$ cases, $96.9 \%$, respectively; $p<0.001)$. There were significantly lower estimated intra-operative blood losses ( $1168 \pm 707$ versus $1520 \pm 845 \mathrm{ml}$, respectively; $p<0.01)$, and related post-operative complications after the introduction of the protocol. The total operative time was around $30 \mathrm{~min}$ shorter $(90.7 \pm 46.9$ versus $117.1 \pm 74.2 \mathrm{~min}$, respectively), but this difference did not reach statistical significance. The length of 
postoperative hospitalization stay was significantly shorter $(5.7 \pm 2.2$ versus $10.9 \pm 14.1$ days, respectively; $p<0.05)$ after the introduction of the protocol.

The clinical characteristics of patients diagnosed with VP before and after implementation of the scanning protocol are presented and compared in Table 2 and Figure 2. The prenatal detection rate of VP increased significantly (9/18 (50\%) cases, versus $29 / 33(87.9 \%)$ cases; $p<0.01)$ and gestational age at diagnosis was significantly earlier $(28.0 \pm 6.4$ versus $32.1 \pm 6.8$ weeks; $p=0.038)$ after the introduction of the protocol. For the neonates, the was a significant reduction in the number of 1 minute Apgar score $\leq 5$ (6/18 33.3\%) cases versus 2/33 (6.1\%) cases; $p<0.05), 5$ minute Apgar score $\leq 5(5 / 18(27.8 \%)$ cases versus $1 / 33(3 \%)$ cases; $\mathrm{p}<0.05)$ and umbilical cord $\mathrm{pH}<7(4 / 18(22.2 \%)$ cases versus $1 / 33(3 \%)$ cases; $p<0.05)$ after the introduction of the protocol. At birth, the newborns initial haemoglobin $(\mathrm{Hb})$ levels were significantly higher (13.7 \pm 3.5 versus16.5 \pm 2.5 ; $p<0.05)$ after the introduction of the targeted protocol. There was only one case of perinatal death due to ruptured VP before the introduction of the protocol.

The false-positive for PAS and VP after the introduction of the protocol were $5 / 37$ cases, $0.1 \%$ versus $3 / 36$ cases, $0.08 \%$, respectively. 


\section{COMMENT}

\section{Principal findings of the study}

The results of present study indicate that the use of targeted scanning protocols for PAS and VP improves the prenatal diagnosis of both of these congenital disorders and improves perinatal outcome. Accurate prenatal diagnosis enables the involvement of MDT and to plan delivery in centres of excellence with access to blood banks, expert surgeons and intensive care for both mothers and their newborns. This reduces the risks of perinatal complications mainly per-operative blood loss and post-operative hospitalization for women with PAS and low Apgar scores and umbilical cord $\mathrm{pH}$ and higher $\mathrm{Hb}$ levels for newborns with VP.

\section{Comparison with existing literature}

The prevalence of both PAS and VP have increased over the last decades due an increase in the numbers of $C D$ and easier access to fertility treatment such as IVF in most regions worldwid. ${ }^{25,26}$ Both are associated with a higher incidence of placenta previa or low-lying placenta and IVF is associated with a higher incidence of $\mathrm{VCl}$ and multiple pregnancies. ${ }^{17,27-30}$ In the present study, we found a similar trends in our population with a progressive increased in the number of singleton pregnancies with a prior CD since 1988 and multiple pregnancies conceived with IVF since. 
There is no universal scanning for PAS and VP and all studies on the prenatal diagnosis of these conditions are from specialist centres. By contrast, there are international screening protocols with standard anatomical views at 11-14 weeks and mid-gestation ultrasound examinations for all the other fetal organs and ultrasound diagnosis of PAS and VP is not routinely taught during ultrasound training courses.

Recent population studies have shown that PAS remains undiagnosed before delivery in half to two-thirds of cases ${ }^{31,32}$ whereas in specialist diagnostic units in the US, around a third of cases of PAS remain undiagnosed during pregnancy. ${ }^{33}$ In a recent systematic review and meta-analysis, we found that ultrasound imaging is highly sensitive and specific in the prenatal diagnosis of PAS when performed by skilled operators, in particular in women presenting with a low-lying placenta/ previa and a history of prior CD. ${ }^{12}$ The pooled performance of ultrasound for the antenatal detection of placenta previa accreta in prospective studies from specialist centres indicates a sensitivity of $97.0 \%(95 \% \mathrm{Cl}, 93.0-99.0)$; specificity of $97.0 \%$ (95\% Cl, 97.0-98.0) and diagnostic odds ratio DOR of $228.5(95 \% \mathrm{Cl}, 67.2-$ 776.9). ${ }^{12}$ The data of the present study indicate that the prenatal detection rate of PAS can be significantly increased by the introduction of a targeted scanning protocol.

VP can be diagnosed prenatally, using combined abdominal and transvaginal ultrasound and colour flow mapping ${ }^{2}$ and the training required to make the diagnosis at the mid-pregnancy scan is simpler than the one require for PAS. The universal screening for VP is an attractive and desirable option to reduce perinatal 
mortality ${ }^{34}$, but published evidence in support of this approach remains limited to data from specialist centres. A recent systematic review including two prospective and six retrospective cohort studies out of which six had a poor methodology including a total of 442,633 women found prenatal detection rates ranging between $53 \%$ to $100 \%{ }^{18}$ Similarly to PAS, the implementation of a targeted scanning protocol improved significantly the prenatal detection rate of VP in our population.

\section{Clinical implications}

Regionalization of care for women in centre of excellence with specialist MDT ${ }^{19,20}$ and the use of a standardized management protocol improves maternal outcome in PAS, particularly in invasive cases. ${ }^{35}$ The impact on maternal and fetal outcomes of multidisciplinary management approaches is directly linked to the accuracy prenatal diagnosis for both PAS and VP. As it is unlikely that universal screening will be implemented for both conditions due to cost and lack of trained ultrasonographers to cover the need of a general population, targeted scanning can provide an alternative option to reduce the impact of these conditions on maternal and fetal outcome.

Targeted scanning PAS should include obstetric risk factors of accreta placentation mainly previous $C D(s)$. This highlights the need for developing training programs and national guidelines for PAS targeted scanning protocols. Women with a prior history of $C D$ and presenting with a placenta previa or low-lying placenta have a risk of placenta previa accreta of $4.1 \%$ after one prior CD and $13.3 \%$ after $\geq 2$ previous CDs and should be reviewed by an experience 
sonographer. The prenatal follow-up of a suspected placenta previa accreta is similar that of a non-accreta placenta previa and will depend mainly on maternal symptoms.

Since appropriate preparation for CD in PAS is crucial for maternal outcomes we have recently introduce a simple calculator for risk assessment (http://www.assafh.org/Pages/PPCalc/index.html). Based on ROC curve, we found that the most effective US criteria for detection of MAP were the presence of the placental lacunae (OR 3.5), obliteration of the utero-placental demarcation (OR 12.4) and placenta previa (OR 10.5). We are aware that there are also sophisticated rule ways of approaching this matter and our model may undergo modification as additional markers may be found, but the simple rule approach is tempting. ${ }^{36}$

The 2014 UK NSC external review found that a targeted screening of pregnancies with at least one high risk factor could reduce perinatal loss rate by as many as 150 cases per year. ${ }^{34}$ Similarly to PAS, the screening for VP could be targeted to women presenting with a low-lying placenta and/or an IVF pregnancy and/or a multiple pregnancy. ${ }^{15,16}$ The combination of these risks factors could identify $90 \%$ of the women at risk of VP and location of the umbilical cord insertion can be easily evaluate on abdominal ultrasound. There is no consensus on the optimal surveillance strategy in women diagnosed with VP. Data from a decision analysis study comparing 11 strategies for delivery timing in a patient with VP found that an elective caesarean delivery between 34 and 36 weeks balances the risk of premature rupture of the membranes and subsequent fetal hemorrhage and 
death versus the risks of prematurity ${ }^{37}$ Antenatal hospitalization to allow for closer surveillance for signs of labor in a unit with appropriate neonatal facilities has also been proposed for all pregnancies presenting with VP from 30-32 weeks of gestation, but the evidence is weak and based on low-quality evidence. ${ }^{2,7}$ Our recent data suggest that consecutive targeted scanning of women with VP combined with its rate of shortening can assist clinicians in identifying those who are at risk for emergent CD. ${ }^{38}$

\section{Strengths and weaknesses}

The main strength of our study is that we have shown for the first time that the implementation of standardised scanning protocol including clinical risk factors and ultrasound features can identify more than $90 \%$ of the cases of PAS or VP prenatally and improve perinatal outcomes for both conditions. The significant increase in the numbers of women with a diagnosis of PAS or VP overtime reflects the increase in the main risk factors i.e. CD and IVF pregnancy rates, for these conditions in our study population, highlighting the increasing need for targeted scanning programs.

There are several limitations to the present study. The retrospective design for the historical cohorts i.e. before implementation of the scanning protocols leads inevitably to missing or incomplete data and an inability to collect all required information. In particular, mode of conception and history of other prior uterine minor surgical procedures such as curettage or endometrial ablation could not be obtained in all cases. Furthermore, the data derived from a facility-based rather 
than a population based registry and were follow-up in a specialist centre with access to both adult and neonatal intensive care. This can explain the low morbidity associated with VP in the historical cohort. In cases PAS, the surgical team was not blinded to the ultrasound findings and the diagnosis of abnormally adherent versus invasive placentation was made by the surgeons during CD. In all cases of abnormal adherence, a conservative management was attempted successfully and no objective histopathological evaluation was obtained.

\section{Conclusion}

Although the current literature lacks confirmatory evidence for the potential benefits of universal prenatal screening for both PAS and VP during the second trimester of pregnancy, the diagnosis of these conditions before delivery is essential for the development of local or regional MDT and management protocols. Identify high groups and targeted scanning is therefore an alternative to universal screening. Our data suggest that a perinatal strategy including standardised targeted scanning protocols, increasing awareness of health care providers of the main risk factors of PAS and VP and training in identifying the ultrasound markers of those abnormalities is associated with improved outcome for both PAS and VP. Such an approach should be considered at regional and national levels and evaluated prospectively. 


\section{REFERENCES}

1. Benirschke K, Burton GJ, Baergen RN. Pathology of the Human Placenta. $6^{\text {th }}$ edition, Springer-Verlag, Berlin, 2012.

2. Silver BM. Abnormal placentation: Placenta previa, vasa previa and placenta accreta. Obstet Gynecol 2015;126:654-68.

3. Royal College of Obstetricians and Gynaecologists. Green-top Guideline No.27: Placenta praevia, placenta praevia accreta and vasa praevia: diagnosis and management. London: RCOG; 2011.

4. Committee opinion no. 529: placenta accreta. Committee on Obstetric Practice. Obstet Gynecol. 2012;120:207-11.

5. Reddy UM, Abuhamad AZ, Levine D, Saade GR; Fetal Imaging Workshop Invited Participants. Fetal imaging: executive summary of a joint Eunice Kennedy Shriver National Institute of Child Health and Human Development, Society for Maternal-Fetal Medicine, American Institute of Ultrasound in Medicine, American College of Obstetricians and Gynecologists, American College of Radiology, Society for Pediatric Radiology, and Society of Radiologists in Ultrasound Fetal Imaging Workshop. J Ultrasound Med. 2014;33:745-57.

6. Wood PL. Vasa praevia and placenta praevia screening in pregnancy. External review against programme criteria for the UK National Screening Committee (UK NSC) [www.screening.nhs.uk/policies];2014. 
7. Society of Maternal-Fetal (SMFM) Publications Committee, Sinkey RG, Odibo AO, Dashe JS. \#37: Diagnosis and management of vasa previa. Am J Obstet Gynecol 2015;213:615-9.

8. Gagnon R. No.231 Guidelines for the management of vasa previa. J Obstet Gynaecol Can. 2017;39:e415-21.

9. Swank ML, Garite TJ, Maurel K, et al; Obstetrix Collaborative Research Network. Vasa previa: diagnosis and management. Am J Obstet Gynecol 2016:215:223.e1-e6.

10. Jauniaux E, Silver RM. Moving from intra-partum to prenatal diagnosis of placenta accreta: a quarter of a century in the making but still a long road to go. BJOG 2017;124:96.

11. Jauniaux E, Savvidou MD. Vasa praevia: more than 100 years in preventing unnecessary fetal deaths. BJOG. 2016;123:1287.

12. Jauniaux E, Bhide A. Prenatal ultrasound diagnosis and outcome of placenta previa accreta after cesarean delivery: a systematic review and meta-analysis. Am J Obstet Gynecol. 2017;217:27-36.

13. Jauniaux E, Collins S, Burton GJ. Placenta accreta spectrum: pathophysiology and evidence-based anatomy for prenatal ultrasound imaging. Am J Obstet Gynecol 2017. doi: 10.1016/j.ajog.2017.05.067.

14. Jauniaux E, Collins SL, Jurkovic D, Burton GJ. Accreta placentation: a systematic review of prenatal ultrasound imaging and grading of villous invasiveness. Am J Obstet Gynecol.2016;215:712-21. 
15. Ruiter L, Kok N, Limpens J, Derks JB, de Graaf IM, Mol B, et al. Incidence of and risk indicators for vasa praevia: a systematic review. BJOG. 2016;123:1278-87.

16. Sullivan EA, Javid N, Duncombe G, et al. Vasa Previa Diagnosis, Clinical Practice, and Outcomes in Australia. Obstet Gynecol. 2017;130:591-8.

17. Jauniaux E, Melcer $Y$, Maymon R. Prenatal diagnosis and management of vasa previa in twin pregnancies: a case series and systematic review. Am J Obstet Gynecol. 2017;216:568-575.

18. Ruiter L, Kok N, Limpens J, Derks JB, de Graaf IM, Mol BW, et al. Systematic review of accuracy of ultrasound in the diagnosis of vasa previa. Ultrasound Obstet Gynecol. 2015;45:516-22.

19. Silver RM, Fox KA, Barton JR, et al. Center of excellence for placenta accreta. Am J Obstet Gynecol 2015;212:561-8.

20. Shamshirsaz AA, Fox KA, Erfani $\mathrm{H}$, et al. Multidisciplinary team learning in the management of the morbidly adherent placenta: outcome improvements over time. Am J Obstet Gynecol. 2017;216:612.e1-612.e5.

21. Oyelese $\mathrm{Y}$, Catanzarite V, Prefumo F, et al. Vasa previa: the impact of prenatal diagnosis on outcomes. Obstet Gynecol 2004;103:937-42.

22. Tovbin J, Melcer Y, Shor S, et al. Prediction of morbidly adherent placenta using a scoring system. Ultrasound Obstet Gynecol 2016;48:504-10.

23. Collins SL, Stevenson GN, Al-Khan A, et al. Three-Dimensional Power Doppler Ultrasonography for Diagnosing Abnormally Invasive Placenta and Quantifying the Risk. Obstet Gynecol. 2015;126:645-53. 
24. Smorgick N, Tovbin $\mathrm{Y}$, Ushakov $\mathrm{F}$, et al. Is neonatal risk from vasa previa preventable? The 20-year experience from a single medical center. J Clin Ultrasound 2010;38:118-22.

25. Vogel JP, Betrán AP, Vindevoghel N, et al; WHO Multi-Country Survey on Maternal and Newborn Health Research Network. Use of the Robson classification to assess caesarean section trends in 21 countries: a secondary analysis of two WHO multi-country surveys. Lancet Glob Health. 2015;3:e260-70.

26. Inhorn MC, Patrizio P. Infertility around the globe: new thinking on gender, reproductive technologies and global movements in the 21st century. Hum Reprod Update. 2015;21:411-26.

27. Ananth CV, Demissie K, Smulian JC, Vintzileos AM. Placenta praevia in singleton and twin births in the United States, 1989 through 1998: a comparison of risk factor profiles and associated conditions._Am J Obstet Gynecol. 2003;188:275-81.

28. Marshall NE, Fu R, Guise JM. Impact of multiple cesarean deliveries on maternal morbidity: a systematic review. Am J Obstet Gynecol. 2011;205:262.e1-8.

29. Klar M, Michels KB. Cesarean section and placental disorders in subsequent pregnancies - a meta-analysis. J Perinat Med. 2014;42:571-83.

30. Jauniaux E, Ben-Ami I, Maymon R. Do assisted-reproduction twin pregnancies require additional antenatal care? Reprod Biomed Online. 2013;26:107-19. 
31. Fitzpatrick KE, Sellers S, Spark P, Kurinczuk JJ, Brocklehurst P, Knight M. The management and outcomes of placenta accreta, increta, and percreta in the UK: a population-based descriptive study. BJOG 2014;121:62-70; discussion 70-61.

32. Thurn L, Lindqvist PG, Jakobsson M, Colmorn LB, Klungsoyr K, Bjarnadottir $\mathrm{RI}$, et al. Abnormally invasive placenta-prevalence, risk factors and antenatal suspicion: results from a large population-based pregnancy cohort study in the Nordic countries. BJOG. 2016;123:1348-55.

33. Bowman ZS, Eller AG, Kennedy AM, Richards DS, Winter TC, 3rd, Woodward PJ, et al. Accuracy of ultrasound for the prediction of placenta accreta. Am J Obstet Gynecol. 2014;211:177 e171-7.

34. Wood PL. Vasa praevia and placenta praevia screening in pregnancy. External review against programme criteria for the UK National Screening Committee (UK NSC) [www.screening.nhs.uk/policies];2014.

35. Shamshirsaz AA, Fox KA, Salmanian B, Diaz-Arrastia CR, et al. Maternal morbidity in patients with morbidly adherent placenta treated with and without a standardized multidisciplinary approach. Am J Obstet Gynecol. 2015;212:218.e1-9.

36. Maymon R, Melcer Y, Pekar-Zlotin M, Shaked O, Cuckle H, Tovbin J. Bedside Risk Estimation of Morbidly Adherent Placenta Using Simple Calculator. Arch Gynecol Obstet, 2018 (In press).

37. Robinson BK, Grobman WA. Effectiveness of timing strategies for delivery of individuals with vasa previa. Obstet Gynecol 2011;117:542-9. 
38. Maymon R, Melcer Y, Tovbin J, Pekar-Zlotin M, Smorgick N, Jauniaux E. The rate of cervical length shortening in the management of vasa previa. JUM, 2017 (in press). 
Table 1. Comparison of the clinical characteristics and outcome of 97 patients diagnosed with placental accreta spectrum (PAS) before and after introduction of the screening protocol (data are presented as mean (SD) or \%).

\begin{tabular}{lccc}
\multicolumn{1}{c}{ Variable } & $\begin{array}{l}\mathbf{2 0 0 4 - 2 0 1 2} \\
(\mathbf{n = 6 5 )}\end{array}$ & $\begin{array}{c}\mathbf{2 0 1 3 - 2 0 1 6} \\
(\mathbf{n = 3 2})\end{array}$ & P value \\
\hline Maternal age (years) & $35.4(4.1)$ & $36.1(3.0)$ & $0.386^{\mathrm{a}}$ \\
BMI (kg/m²) & $31.5(6.1)$ & $32.2(5.3)$ & $0.643^{\mathrm{a}}$ \\
& & & \\
Obstetric history & & & \\
Gravidity [median (interquartile range)] & $4(3-5)$ & $4(3-5)$ & $0.809^{\mathrm{a}}$ \\
Parity [median (interquartile range)] & $2(1-3)$ & $2(1-3)$ & $0.951^{\mathrm{a}}$ \\
Previous CD & $59(90.8 \%)$ & $32(100 \%)$ & $0.173^{\mathrm{c}}$ \\
Placenta previa & $39(60 \%)$ & $20(62.5 \%)$ & $1.0^{\mathrm{c}}$ \\
& & & \\
Current pregnancy & & & \\
Prenatal diagnosis & $28(43.1 \%)$ & $31(96.9 \%)$ & $<0.001^{\mathrm{c}}$ \\
Gestational age at diagnosis (weeks) & $34.1(4.1)$ & $33.9(4.3)$ & $0.825^{\mathrm{a}}$ \\
& & & \\
Surgical characteristics & & & \\
Elective CD & $48(73.8 \%)$ & $24(75.0 \%)$ & $1.0^{\mathrm{c}}$ \\
General anesthesia & $38(58.5 \%)$ & $19(59.4 \%)$ & $0.594^{\mathrm{d}}$ \\
Low segment transverse uterine incision & $52(80.0 \%)$ & $24(75.0 \%)$ & $0.606^{\mathrm{c}}$ \\
Cesarean hysterectomy & $23(35.9 \%)$ & $11(34.4 \%)$ & $0.956^{\mathrm{d}}$ \\
Surgery duration (min) & $117(74)$ & $90(46)$ & $0.135^{\mathrm{a}}$ \\
Estimated blood loss (ml) & $1520(845)$ & $1168(707)$ & $<0.01^{\mathrm{a}}$ \\
Early composite morbidity & $21(32.3 \%)$ & $5(15.6 \%)$ & $0.081^{\mathrm{d}}$ \\
Duration of hospitalization (days) & $10.9(14.1)$ & $5.7(2.2)$ & $0.024^{\mathrm{a}}$ \\
& & & \\
Laboratory findings & & & \\
Preoperative hemoglobin (mg/dL) & $10.9(1.1)$ & $11.2(1.4)$ & $0.264^{\mathrm{a}}$ \\
Postoperative hemoglobin (mg/dL) & $8.8(1.4)$ & $10.1(1.6)$ & $<0.001^{\mathrm{a}}$ \\
Hemoglobin decrease (mg/dL) & $2.1(1.5)$ & $1.3(1.5)$ & $<0.01^{\mathrm{a}}$ \\
& & & \\
No of blood products transfusion & & & \\
Intraoperative packed red blood cell transfusion units & $4.9(6.6)$ & $2.3(2.6)$ & $0.064^{\mathrm{b}}$ \\
Intraoperative fresh frozen plasma transfusion units & $2.5(4.3)$ & $1.3(1.9)$ & $0.449^{\mathrm{b}}$ \\
Intraoperative platelets transfusion units & $1.3(5.4)$ & $0.5(1.9)$ & $0.581^{\mathrm{b}}$ \\
Intraoperative cryoprecipitate transfusion units & $2.1(4.3)$ & $0.6(2.1)$ & $0.055^{\mathrm{b}}$ \\
Postoperative packed red blood cell transfusion units & $1.3(3.2)$ & $0.06(0.3)$ & $<0.05^{\mathrm{a}}$ \\
Total blood products transfusion units & $11.9(20.3)$ & $4.5(6.7)$ & $<0.05^{\mathrm{a}}$ \\
& & &
\end{tabular}


Gestational age at delivery (weeks)

$36.0(3.0)$

$2664(750)$

$8.4(1.4)$

$9.7(0.6)$

7.3(0.07)
$36.6(2.3)$

$0.319^{a}$

$2839(528)$

$0.242^{\mathrm{a}}$

$8.8(0.5)$

9.8(0.4)

$0.167^{\mathrm{a}}$

7.3(0.06)

$0.129^{a}$

Umbilical cord $\mathrm{pH}$

${ }^{\text {aT }}$ Two tailed t-test; ${ }^{b}$ Mann-Whitney rank test; 'Fisher's Exact test; ${ }^{d}$ Pearson ChiSquare test ${ }^{\S}$ Defined as the occurrence of one or more of the following during hospitalization period: endometritis, wound infection/hematoma, pelvic abscess, relaparotomy, thromboembolism, coagulopathy, ileus and injuries to adjacent organs. $C D=$ Caesarean delivery. 
Table 2. Comparison of characteristics of 51 patients diagnosed with vasa previa before and after introduction of the screening protocol (data are presented as mean (SD) or \%).

${ }^{\text {aT }}$ wo tailed t-test; ${ }^{\text {b}}$ Fisher's Exact test. $\mathrm{CD}=$ Caesarean delivery; $\mathrm{ART}=$ Assisted reproductive technologies

Variable

Variable

Maternal age (years)

Obstetric history

Gravidity [median (interquartile range)]

Parity [median (interquartile range)]

\section{Current pregnancy}

Mode of conception

Spontaneous

ART

Singletons

Twins

Prenatal diagnosis

Placenta previa

Gestational age at diagnosis (weeks)

CD

Elective CD

\section{Neonatal outcome}

Gestational age at delivery (weeks)

Birth weight (gram)

Apgar score at 1 minute

Apgar score at 5 minute

No of 1 minute Apgar score $\leq 5$

No of 5 minute Apgar score $\leq 5$

Umbilical cord $\mathrm{pH}$

No of umbilical cord $\mathrm{pH}<7$

Newborn initial hemoglobin $(\mathrm{mg} / \mathrm{dL})$

Immediate newborn resuscitation

Immediate newborn Intubation

Blood products transfusion

Admission to neonatal intensive care unit

Perinatal death

Duration of newborn hospitalization (days)
1988-2007

$(\mathrm{n}=18)$

32.2(4.8)

$2(1-3)$

$1(0-1)$

2(1-3)

$0.591^{\mathrm{a}}$

$1(0-1)$

$0.552^{\mathrm{a}}$ $(\mathrm{n}=33)$
2008-2016 P value

$33.8(4.9) \quad 0.252^{\mathrm{a}}$

$\begin{array}{ccc}9(50.0 \%) & 15(45.5 \%) & 0.778^{\mathrm{b}} \\ 9(50.0 \%) & 18(54.5 \%) & \\ 15(83.3 \%) & 28(84.8 \%) & 1.0^{\mathrm{b}} \\ 3(16.7 \%) & 5(15.2 \%) & \\ 9(50 \%) & 29(87.9 \%) & <0.01^{\mathrm{b}} \\ 5(27.8 \%) & 16(48.5 \%) & 0.234^{\mathrm{c}} \\ 32.1(6.8) & 28.0(6.4) & 0.038^{\mathrm{a}} \\ 16(88.9 \%) & 32(97.0 \%) & 0.282^{\mathrm{b}} \\ 4(22.2 \%) & 14(42.4 \%) & 0.222^{\mathrm{b}}\end{array}$

\begin{tabular}{ccc}
$36.3(2.2)$ & $36.3(1.9)$ & $0.939^{\mathrm{a}}$ \\
$2684(652)$ & $2666(546)$ & $0.917^{\mathrm{a}}$ \\
$5.9(4.0)$ & $8.4(1.9)$ & $<0.005^{\mathrm{a}}$ \\
$6.6(3.8)$ & $9.4(1.4)$ & $<0.001^{\mathrm{a}}$ \\
$6(33.3 \%)$ & $2(6.1 \%)$ & $<0.05^{\mathrm{b}}$ \\
$5(27.8 \%)$ & $1(3.0 \%)$ & $<0.05^{\mathrm{b}}$ \\
$7.1(0.1)$ & $7.3(0.1)$ & $<0.05^{\mathrm{a}}$ \\
$4(22.2 \%)$ & $1(3.0 \%)$ & $<0.05^{\mathrm{b}}$ \\
$13.7(3.5)$ & $16.5(2.5)$ & $<0.05^{\mathrm{a}}$ \\
$4(22.2 \%)$ & $6(18.2 \%)$ & $0.727^{\mathrm{b}}$ \\
$4(22.2 \%)$ & $4(12.1 \%)$ & $0.429^{\mathrm{b}}$ \\
$5(27.8 \%)$ & $6(18.2 \%)$ & $0.488^{\mathrm{b}}$ \\
$8(44.4 \%)$ & $18(54.5 \%)$ & $0.565^{\mathrm{b}}$ \\
$1(5.5 \%)$ & $0(0 \%)$ & $0.353^{\mathrm{b}}$ \\
$6.7(4.4)$ & $7.9(10.3)$ & $0.689^{\mathrm{a}}$ \\
\hline
\end{tabular}




\section{Figure legends}

Figure 1. Number of $(A)$ deliveries per year during the study period; $(B)$ percentage of caesarean deliveries and (C) percentage of pregnancies resulting from IVF per year.

A
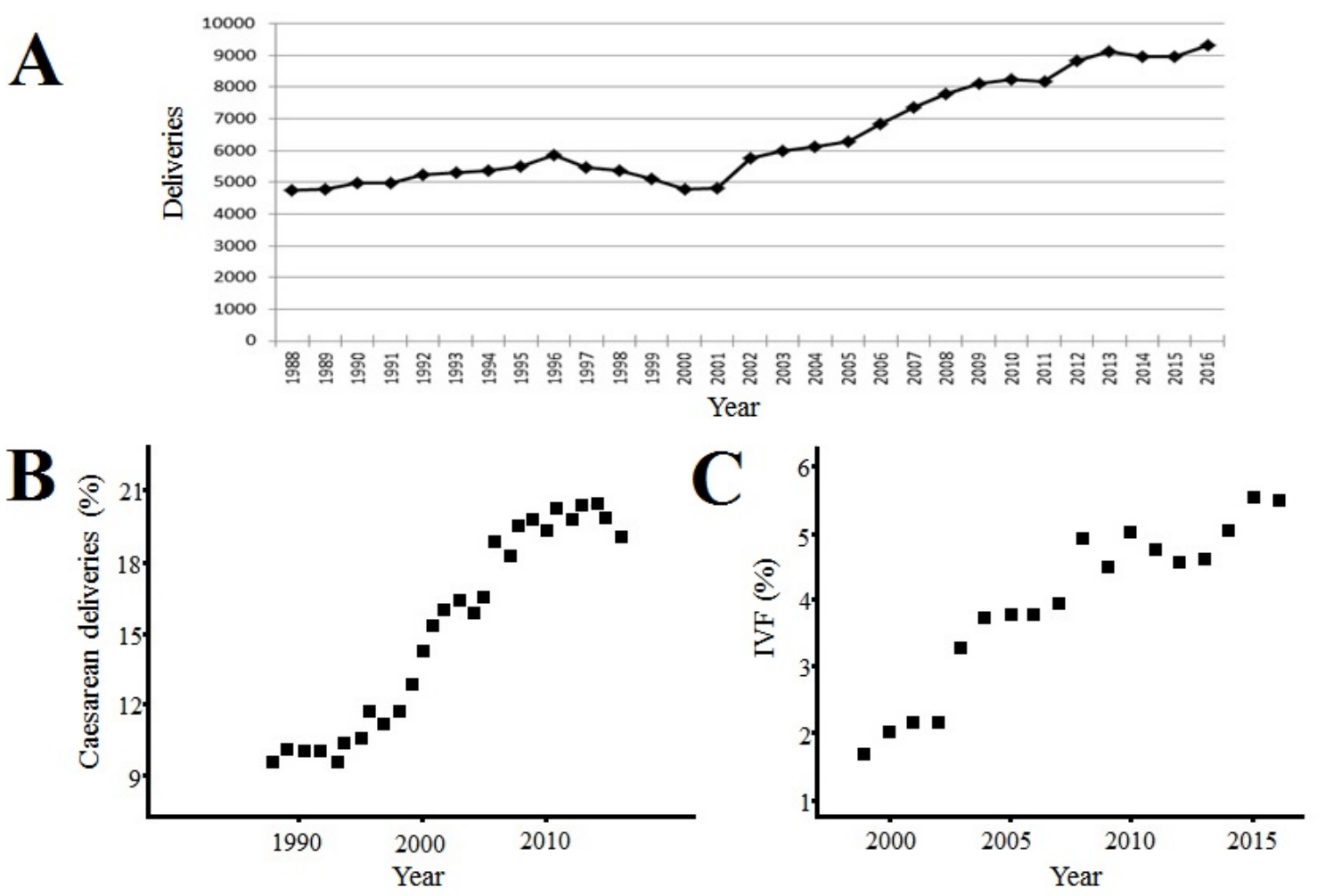
Figure 2. Incidence (per 1000 deliveries) and prenatal detection rate of placenta accreta spectrum (PAS) and vasa previa (VP) during study periods.

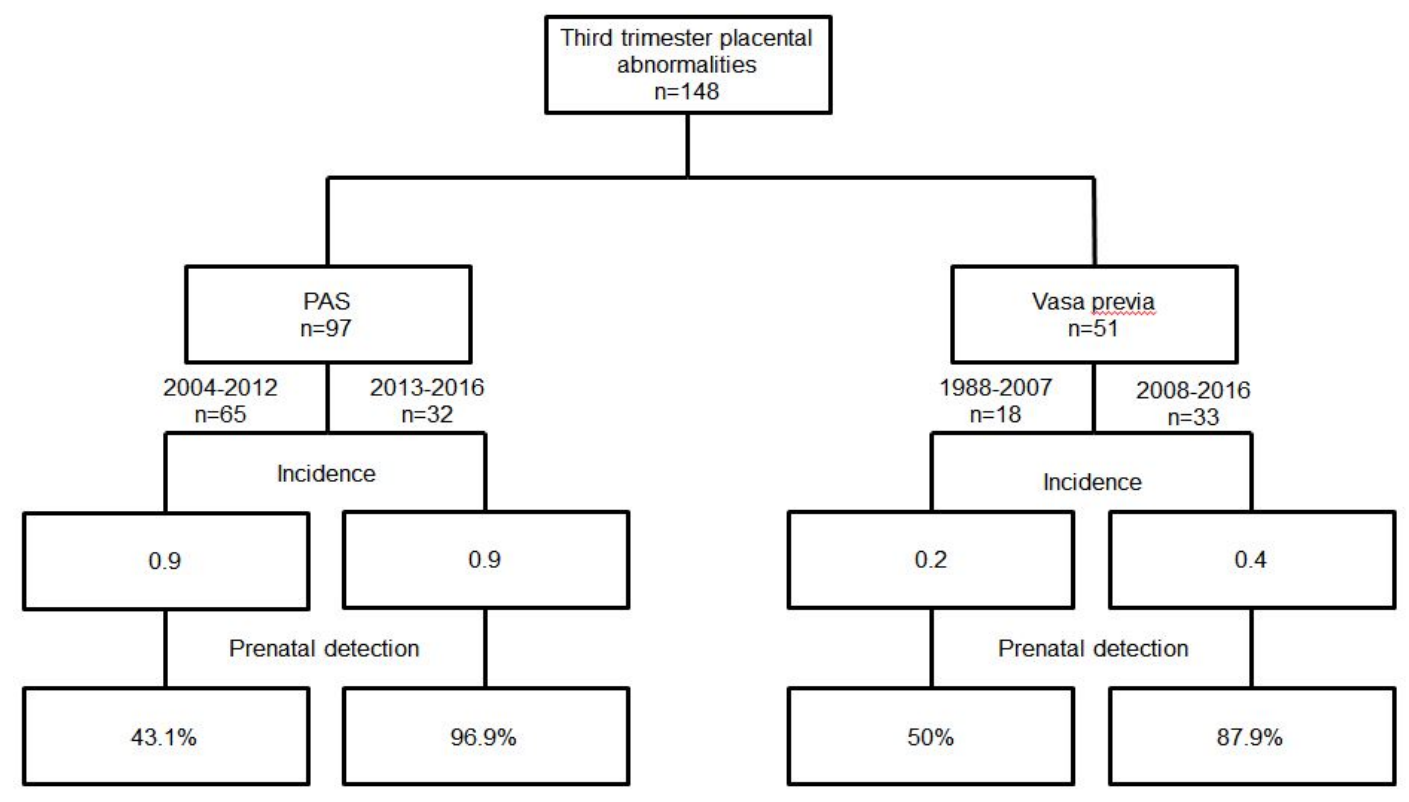

